\title{
Morfosintaxis del waunana a partir de un texto tradicional ${ }^{1}$
}

\author{
José Manuel Murillo Miranda ${ }^{2}$ \\ Universidad Nacional, Costa Rica
}

\begin{abstract}
resumen
Es una descripción general de los aspectos morfológicos y sintácticos generales de la lengua waunana, a partir de un análisis hecho a una historia tradicional. El estudio se enmarca dentro del trabajo de recopilación y revitalización llevado a cabo por el Programa de Lenguas Indígenas de la Baja Centroamérica.
\end{abstract}

\begin{abstract}
This is a general description of the main morphological and syntactic phenomena of Waunana language, as an outcome of analyzing a traditional story. This study is part of the collection and revitalization work conducted by the Program of Indigenous Languages of the Lower Central America.
\end{abstract}

Palabras clave: waunana, lenguas chocó, gramática, Panamá, lenguas amerindias

Keywords: Waunana, Choco languages, grammar, Panama, Amerindian languages

1 Versión revisada de la ponencia leída en el marco del I Simposio Internacional del Programa de Lingüística Centroamericana (PROLINCA) «Centroamérica: un microcosmo lingüístico, llevado a cabo los 7 y 8 de mayo 2012, en la ciudad de Heredia, en el campus Omar Dengo de la Universidad Nacional de Costa Rica. Recibido: 12 de diciembre de 2011; aceptado: 2 de febrero de 2012. 2Escuela de Literatura y Ciencias del Lenguaje. Correo electrónico: jo.m.uri@ hotmail.com

$$
L_{\text {etras }} 51 \text { (2012), ISSN 1409-424X } 59
$$




\section{Introducción}

Este trabajo es una aproximación inicial a la morfología y a la sintaxis de la lengua waunana, lengua chocó hablada en la provincia de Darién, en Panamá. Forma parte de una investigación más amplia dividida en dos etapas, cuyo resultado final será una gramática descriptiva. El documento se organiza en dos secciones: la primera corresponde a algunos datos generales de la lengua waunana, su ubicación geográfica, su estado de vitalidad y otros datos etnográficos; la segunda corresponde a la descripción de las propiedades gramaticales de morfología y sintaxis que se pueden determinar con base en el análisis de una historia tradicional.

\section{La lengua waunana}

\section{Ubicación geográfica y genética}

La lengua waunana, woun meu [Çwow) Èmew] pertenece a la familia chocó, cuyos miembros son el emberá y el waunana; se ubica en las provincias de Darién, Panamá, y en el departamento de Chocó en Colombia; en este último la lengua habladan unas 3000 personas. La línea fronteriza entre estos dos países no tiene incidencia en cuanto a la variación diatópica; las diferencias geográficas se dan en relación con tierras altas (dusĩ pien) y tierras bajas (du charpien). Puede que las diferencias entre ambas variantes están relacionadas con el vocalismo, concretamente en la zona medio-alta del espectro vocálico.

En Panamá, la lengua la hablan aproximadamente unas diez mil personas a lo largo del territorio; sin embargo, la mayoría de hablantes se ubica en la Comarca Emberá-Wounaan, localizada en la provincia del Darién ${ }^{3}$. Esa comarca está dividida en dos: la Zona

2 Para los datos sobre la ubicación y distribución de esta lengua, se parte de la información que ofrece M. Paul Lewis (ed.), y en particular de los mapas «Ubicación de los waunana en Colombia» y «Ubicación de la comarca emberá-wounaan», datos extraídos, a su vez, de su obra del Instituto Lingüístico de Verano: Woun Meu. Ethnologue: Languages of the World, 16.a ed. (Dallas, TX: SIL International, 2009); están disponibles también en versión digital: 〈http://www.ethnologue.com>. 
1 y la Zona 2; esta última habitada por los emberá. En la 1 hay seis comunidades wounaan: dos comunidades en el río Membrillo: Canaán, Sinaí; en la desembocadura del río Tupiza está la comunidad de Belén; sobre el río Tuira está la comunidad de Capetí, Vista Alegre y Aruza. En la zona de tierras colectivas (territorios de reducida extensión al externo de la comarca) las comunidades son: Puerto Lara, Pueblo Nuevo, Chuletí (en la ribera del río Balsa), Anayansi (en la ribera el río Pihuila) y Boca de Mono; sobre la costa se encuentra, por la ribera del río Taimatí, la comunidad de Cémaco. En la costa del río Congo, en la ribera de río Sucio, está la comunidad de Caña Blanca; cerca de la frontera colombo-panameña, en la ribera del río Jaqué está la comunidad de Bidoquera; en la zona interprovincial Panamá-Darién, en la ribera del río Majé está la comunidad del mismo nombre; y en la ribera de los ríos Platanares y río Hondo se encuentran las comunidades del mismo nombre, respectivamente. Hacia el lago Gatún, Provincia de Panamá, hay dos comunidades minúsculas. Finalmente, hay pequeñísimas colonias en la ciudad de Panamá, así como en otros lugares del país.

La organización interna de los waunanas la determinan las disposiciones territoriales vigentes en Panamá; a saber: un cacique elegido mediante voto universal en la comarca, y otro proveniente de las tierras colectivas; en cuanto al primero, no siempre es un waunana, porque depende de la totalidad de la población y en la comarca, la mayoría pertenece a la etnia emberá. El cacique de tierras colectivas lo eligen dirigentes representantes de cada una de las comunidades, mediante el Congreso Wounaan. El cacique de la comarca es electo por un período de cuatro años, reelegible, mientras que el de tierras colectivas lo es por tres años, igualmente reelegible. Los dirigentes comunales son electos por períodos de dos años, reelegibles, aunque tambiénrevocables.

\section{Grado de vitalidad/declinación}

Los propios hablantes manifiestan que en todas las comunidades la lengua está en peligro de perderse, pues ya no lo hablan los 
jóvenes. A pesar de que existe una Dirección de Educación Intercultural Bilingüe del Ministerio de Educación de Panamá, la educación en primaria y secundaria se desarrolla en español; no se imparte la lengua en las escuelas de las comunidades waunanas y, en general, hay escaso apoyo institucional o voluntad política para proteger las lenguas indígenas de Panamá. Sin embargo, se reconocen tres comunidades como representativas de una creciente preocupación por mantener la lengua; estas son Puerto Lara, Sinaí y Vista Alegre.

La clasificación de Bauman ${ }^{4}$ para analizar el estado en que se encuentran las lenguas indígenas con respecto al inglés en Estados Unidos, plantea las siguientes categorías: 1. florecientes, 2. duraderas, 3. declinantes, 4. obsolescentes, y 5. extintas. Entre los criterios para la clasificación están la edad de los hablantes y su grado de bilingüismo (+), la proporción de hablantes con respecto al total de la población $( \pm)$, fluidez de los hablantes jóvenes $( \pm)$, grado de preferencia por la lengua materna $( \pm)$, grado de bilingüismo $( \pm), y$ capacidad de adaptación de la lengua a una cultura cambiante (-). Los valores que aparecen en paréntesis provienen de la observación realizada en la comunidad de Puerto Lara (Darién) durante la primera visita del proyecto sobre el waunana. Según se desprende de esos valores, el waunana se encuentra, en esta comunidad específica, en estado de duradera. Sin embargo, hay un estado de alerta, pues como se desprende del valor asignado a los parámetros de fluidez de los hablantes jóvenes, grado de preferencia por la lengua materna y grado de bilingüismo, la tendencia subyacente es que las nuevas generaciones ya están mostrando cierta preferencia por el español.

\section{Estudios previos}

Esta lengua no cuenta con una gramática detallada con las características de las que elabora el programa. Los materiales publicados

4 James J. Bauman, A Guide to Issues in Indian Language Retention (Washington: Center for Applied Linguistics, 1980). 
son escasos ${ }^{5}$. Hay un vocabulario trilingüe woun meu-español-epena pedee $^{6}$, una gramática pedagógica incompleta ${ }^{7}$ y un estudio sobre aspectos del discurso ${ }^{8}$. Binder ${ }^{9}$ indica que esa lengua posee veinte fonemas consonánticos (la serie oclusiva sonora /b/, /d/, /g/; las oclusivas sordas /p/, /t/, /k/ y ///; la serie aspirada sorda /pH/, /tH/ y /kH/; una africada $/ \mathrm{tS} /$; dos vibrantes $/ \mathrm{R} / \mathrm{y} / \mathrm{r} / ;$ dos nasales $/ \mathrm{m} / \mathrm{y} / \mathrm{n} / ;$ dos fricativas /s/ y /h/; una lateral /l/, y dos deslizantes /y/ y /w/) y dieciséis fonemas vocálicos divididos en dos grandes series: una serie oral (con las anteriores /i/, /I/, /e/; la central /a/; las posteriores no redondeadas $/ \mu /, / \mathrm{F} /$ y las posteriores redondeadas $/ \mathrm{u} /, / \mathrm{U} / \mathrm{y} / \mathrm{o} /$ ) y una serie nasal más reducida $((/ /),(\mathrm{e}) /,(\mathrm{a}) /, / \mu) /, /(\mathrm{u}) / \mathrm{y} /(0) /))$. No toma la cantidad como un valor fonológico. Aparte de esto, existen algunas publicaciones vernaculares, tanto de manuales de lectoescritura como de cuentos y otros tópicos no lingüísticos, especialmente de orientación religiosa ${ }^{10}$.

\section{Texto waunana}

El texto de donde se tomaron los ejemplos es una historia tradicional (ver anexo). Relata una serie de sucesos que ocurren en una noche y afectan a dos jóvenes y sus novias, a un viejo brujo y a su esposa. En primer lugar se presenta el texto completo en lengua waunana y luego la traducción libre al español. No se ha incluido el texto transmorfemizado debido a su extensión. La historia fue narrada por Diego Upúa; la

5 Existe una gramática de esta lengua, la de Jacob A. Loewen (1954). Se encuentra microfilmada en la biblioteca de la Washington State University.

6 Ronald E. Binder, Phillip L. Harms y Chindío Peña Ismare, Vocabulario ilustrado, tomo 2: Wounmeu - Español - Epena Pedee (Bogotá: Asociación Instituto Lingüístico de Verano, 1995).

7 Micaela Sánchez A. y Olga Castro G., Una gramática pedagógica del waunana: primera parte. Lenguas de Panamá, 3 (Panama: Instituto Lingüístico de Verano: 1977).

8 Ronald E. Binder, «Thematic Linkage in Waunana discourse», en Robert E. Longacre y Frances Woods (eds.), Discourse Grammar: Studies in Indigenous Languages of Colombia, Panama, and Ecuador (vol. 2) (Dallas, TX: Summer Institute of Linguistics and the University of Texas at Arlington, 1977) 159-190.

9 Ronald E. Binder y Kathleen P., «Fonología waunana»,»en Patricia Baptista (red.). Lenguas de Panamá. Tomo I. Sistemas fonológicos. Instituto Lingüístico de Verano e Instituto Nacional de Cultura (Panamá: Imprenta de la Nación, 1974), 71-94.

10 Iglesia Evangélica Unida, Negöon meu k'augwia jũrr maach meujã k'augtarrau. Tomos 1 y 2 (Panamá, 2007). 
transcripción, transmorfemización y traducción se hicieron con la colaboración del pastor Vallarino Cheucarama. Ambos son miembros de la comunidad de Puerto Lara. La presentación de los ejemplos se realiza mediante transcripción ortográfica según el alfabeto waunana avalado por el Ministerio de Educación de Panamá. Solo unos pocos ejemplos, específicamente en el apartado dedicado al orden de palabras en el sintagma nominal, corresponden a cuestionarios independientes.

\section{Aspectos morfológicos}

En esta sección se hace un recuento de las características morfológicas de la lengua, según se desprenden del texto. La sección se divide en morfología nominal, morfología verbal, morfología de los adjetivos, adverbios, adposiciones y otras clases menores.

\section{Dimensión nominal}

Sustantivos

Los sustantivos en waunana son una clase de palabras referidas a entidades de todo tipo, tanto animadas ( köpurr 'sapo') como inanimadas ( $d i$ 'casa'), a humanos (hõre 'gente', $h{ }_{-} i$ 'mujer', chaai 'niño', jooi 'anciano'), animales ( $k u) s$ 'cerdo'), plantas (jarb_ _ palo de achiote'), seres mitológicos (Alpat 'demonio', hewandam 'dios'), partes del cuerpo ( $p$ 'etk'ar 'costilla', $b \downarrow$ ' 'pie, pierna', $t$ 'ããne 'corazón'), cuerpos celestes (heedauha 'sol', hedauark 'luna'), etc. La mayoría de los sustantivos están compuestos por raíces simples ( $k$ 'aper 'compañero'), si bien se han dado con formas compuestas ( $d i$ 'casa' $+b \downarrow_{-}$'pie' $\rightarrow d i b \downarrow_{\text {_ }}$ 'horcón') y un caso de derivación ( $k a \tilde{i}$-tar dormir-nmlz 'el que duerme'). También son evidentes casos de hispanismos, como nobia, bieja, brujo, etc. Los sustantivos no tienen flexión de género, pero sí de número, que se realiza mediante los sufijos $-n$ y - $(n) a a n$; el primero tiende a añadirse a sustantivos terminados en $-r($ bẽnk $\sqrt{ }) r$ 'brujo' $+-n \rightarrow$ bẽnk $\sqrt{ }) n$ 'brujos', $n e$ per 'demonio' $+-n \rightarrow$ nepen 'demonios', ãt' ârr 'gallina' $+-n \rightarrow$ ât'ãn 'gallinas') y a sustantivos terminados en vocal $-i$ o en el diptongo -ie (emk'ooi 'hombre' $+-n \rightarrow$ emk'ooin 'hombres', jooi 'viejo, ancestro' 
$+-n \rightarrow$ jooin 'viejos, ancestros', nemchai 'pájaro' $+-n \rightarrow$ nemchain 'pájaros', + beermie 'contador' - $n \rightarrow$ beermien 'contadores' ), el segundo alomorfo (n)aan se añade a sustantivos terminados en otras consonantes $(k u) s$ 'cerdo'+-naan $\rightarrow k \mathrm{u}$ )snaan, mök 'piedra' +-naan $\rightarrow$ möknaan 'piedras', dusig 'río' + -naan $\rightarrow$ dusignaan 'ríos', woun 'persona' + -aan $\rightarrow$ wounaan 'personas') y otras vocales ( $b{ }^{\prime}$ 'pie' + -naan $\rightarrow$ bınaan 'pies', hũa 'mano'+ -aan $\rightarrow$ hũaan). El sustantivo saak 'perro' tiene su plural irregular (saakien 'perros', por analogía con beermie), lo mismo que $d i$ 'casa' $\rightarrow$ dinaan 'casas'; la forma dënnaan 'padres' es pluralia tantum. Los sustantivos no contables, como $d u$ 'agua' no reciben marcación de plural, como tampoco reciben marcación de pluralidad los singularia tantum het'a 'cielo', jep 'tierra', hewandam 'dios', heedauha 'sol', hedauark 'luna' у $p$ 'u 'viento'.

\section{Pronombres}

El sistema de pronombres personales del waunana incluye tres personas con distinción de plural y singular, con la distinción inclusivo-exclusivo de la primera persona plural. Los pronombres se hallan divididos en dos series: la serie ergativa y la serie absolutiva. El cuadro 1 muestra el paradigma pronominal.

\section{Cuadro 1. Paradigma pronominal del waunana}

\begin{tabular}{|l|l|l|l|}
\hline & & Ergativo & Absolutivo \\
\hline \multirow{5}{*}{ SG } & 1 & mиa & $m \sqrt{ }$ \\
& 2 & pua & $p \sqrt{ }$ \\
& 3 & hichdëu & hich \\
& 1 excl & maachdëu & maach \\
& 1 incl & marĩu & mãre \\
pl & pãarau & pãar \\
& 3 & hamachdiu & hamach \\
\hline
\end{tabular}


Determinantes

Los determinantes muestran dos grados de distancia del hablante y una distinción de número (véase el cuadro 2).

\section{Cuadro 2. Determinantes del waunana}

\begin{tabular}{|l|l|l|}
\hline Grado de distancia & $\mathbf{1}$ & $\mathbf{2}$ \\
\hline Singular & $m \downarrow g$ & $j \tilde{a} g$ \\
Plural & $m \downarrow \_\mathrm{k} \downarrow \_n$ & $j \tilde{a} k \downarrow n$ \\
\hline
\end{tabular}

Numerales

El sistema de numerales tradicional está cayendo en desuso y lo va sustituyendo el español. Se basa en dos maneras de contar: con los dedos y con el cuerpo. Con los dedos llega hasta veinte y con el cuerpo, hasta cien. La cuenta con los dedos se realiza con base en los numerales hãp 'uno', numi 'dos, t'ãrjüp 'tres', jayap 'cuatro' y hua hãp 'cinco' (lit. mano uno 'una mano'). La cuenta con el cuerpo toma como base la palabra mõrr mõnã [mõne mõnãp] 'lit. cuerpo' con el significado de 'veinte'. En el cuadro 3 se muestran ambas formas. El sistema es quinario.

\section{Cuadro 3. Numerales básicos y formas de contar del waunana hasta 100}

\begin{tabular}{|c|c|}
\hline hãp & 1 \\
\hline numi & 2 \\
\hline$t^{\prime} \tilde{a} r j u \tilde{u} p$ & 3 \\
\hline jayap & 4 \\
\hline hua hãp & 5 \\
\hline hua hãp awie hãp & $6(5+1)$ \\
\hline hиa hãp awie numi & $7(5+2)$ \\
\hline hua hãp awie t'ãrjūp & $8(5+3)$ \\
\hline hua hãp awie jayap & $9(5+4)$ \\
\hline hиа питi & 10 (mano dos) \\
\hline
\end{tabular}




\begin{tabular}{|l|l|}
\hline mõn hãp & 20 \\
\hline mõn hãp awie hua numi & $30(20+10)$ \\
\hline mõne numi & $40(20 * 2)$ \\
\hline mõne numi awie hua numi & $50(40+10)$ \\
\hline mõn t'ãrjūp & $60(20 * 3)$ \\
\hline mõn t'ãrjūp awie hua numi & $70(60+10)$ \\
\hline mõne jayap & $80(20 * 4)$ \\
\hline mõne jayap awie hua numi & $90(80+10)$ \\
\hline mõne jayap awie mõn hãp & $100(80+20)$ \\
\hline
\end{tabular}

Además de los cardinales, también están los ordinales, creados con la adición del sufijo -(a)m al numeral cardinal, como t’ãrjüp + -am $\rightarrow$ t'ãrjüpam 'tercero', hua hãp awie numi $+-a m \rightarrow$ hua hãp awie numim 'séptimo'. Son formas irregulares nacharam 'primero' y nacharam gare 'segundo'. En cuanto a los partitivos, solo se ha encontrado hãar 'medio', como en bık'ier hãar 'media yuca'.

Finalmente, la lengua waunana presenta un artículo (1), un morfema libre de diminutivo (2), un marcador anafórico (3), cuantificadores (4) e indefinidos (5).

chi hewaan

art joven

'El joven'

hu) ãn jua dam

vieja mano dim

'manita de la vieja'

Petaadi-ji-m di $\quad h \downarrow_{\text {_taag, }}$ waid $V_{-b a a d}$ dib $\sqrt{ }$ gaai, mag irse-pdo.SinG-dcl casa arriba trepar-inc horcón en dib $\sqrt{ }$ gaai waid $\sqrt{ }$-wie

anf horcón en trepar-ant

'Se fue arriba de la casa, empieza a trepar por el horcón; cuando ya había trepado por ese horcón (...) 
Tönwe $m \downarrow$ chaai-n

Todos 1Sing hijo-pl

'Todos mis hijos'

Haũrag $m \downarrow$ kod-naan

Alguno 1sG primo-pl

'Algunos primos míos'

\section{Dimensión verbal}

Según los datos extraídos del texto, la mayor parte de las raíces verbales son simples y tienden a construirse en una o dos sílabas, como en ma-kim ir-inm 'ya voy', $p \downarrow_{-}$rau-wie agarrar-ant 'hubo agarrado', waid $\sqrt{ }$-wie trepar-ant 'hubo trepado'; también existen casos de raíces más largas, como en k'anitoga-ji-m apestar-pdo.SinG-dcl 'apestaba', haup\_abaje-ji-m abandonar-pdo.SinG-dc1 'abandonó', $j \downarrow$ chpieje-ji-m-ta poner-pdo.SinG-dc1-foc 'puso'. El ejemplo (6) muestra el caso de una raíz compuesta por un adjetivo hispano $p e-$ gaa 'pegado, borracho' máseladverbio pai 'medio'. Otros ejemplos de hispanismos como raíces verbales son: rondaan 'vigilar' < rondar, hacee 'hacer' < hacer, regaa 'esparcirse, dispersarse' < regar, celebraa 'celebrar' < celebrar, calmaa 'calmarse' < calmar, mowee 'moverse' < mover, usaa 'usar' < usar. También es posible encontrar casos de raíces verbales que sufren variaciones debidas a las categorías de TAM en las que se encuentren flexionadas, como en $\mathrm{ma}-\mathrm{m}$ ir-dcl 'va' y peta-ji-m ir-pdo-dcl 'fue'.

Mamagsiine jööi pegaapai trago-o, do botella-u en ese momento viejo medio pegado.prs trago-ins dos botella-ins pegaapaiaade-ji-m

medio pegado-pdo.SinG-dcl

En ese momento el viejo se medio emborracha con el trago, con dos botellas se medio emborrachó' 
Las categorías verbales detectadas incluyen la distinción temporal entre el presente, cuya marca es $\varnothing$ (7), (8), (9) y (10), un pasado singular (11) y otro plural (12), un anterior (13), (15) y (16), y un futuro (14); también se encuentra la distinción aspectual de habitual (17); además, aparentemente hay un sufijo para la fase incoativa (18). En cuanto a la modalidad, se ha encontrado un sufijo para la modalidad imperativa con distinción de número (- $b a$ para el singular (16) y -t'at(a) para el plural (19)) y un sufijo - $m$ que marca la modalidad declarativa (Sánchez y Castro, 1977: 25-26) y la distingue de la interrogativa, (7) y (8). Sin embargo, este sufijo declarativo se restringe al presente (al parecer tampoco en todos los casos (9) y (13)), al habitual (17) y al pasado (11), (12), (15) y (18). La negación se codifica como sufijo verbal homófono del imperativo (20). El cuadro 4 resume los sufijos verbales encontrados:

(7) ¿Kanjawia $\downarrow_{\text {_ }}$ k'amiiu si?

Por qué 2sG hediondo estar.prs

¿Por qué está hediondo?'

(8) Hichita poorpam si-m-ta

Verdad dañado estar-dcl-foc

'En verdad está dañado'

(9) Tööd huk'arii tööd weeo

Toldo suavecito toldo abrir.prs

'Abre el toldo muy suave'

(10) jãkwoun mag uru-m, uru-m, uru-m

esa persona anf venir.prS-dcl venir.prS-dcl venir.prS-dcl

'Esa persona viene'

11 Sánchez y Castro denominan este sufijo imperativo de objetivo. Sin embargo, en el texto analizado no se han encontrado contextos imperativos que contengan este morfema.

12 Para Mortensen, la lengua emberá, hermana del waunana, posee también un sufijo declarativo - $a$. A diferencia del waunana, el sufijo - $a$ del emberá también se marca en el futuro, en las formas de los aspectos habitual, perfectivo e imperfectivo. Además, tiene el papel de indicar que la información proporcionada por el hablante es cierta o tomada como cierta. Lo anterior hace pensar que el papel del sufijo declarativo $-m$ está en franca decadencia. Charles A. Mortensen. A Reference Grammar of the Northerm Emberá Languages (Dallas: SIL International, 1999). 
(11) $\quad p \downarrow_{-} j \downarrow_{-} r p \downarrow_{-} i-j i-m$ neper-au

2sG espantar-pdo.SinG-dcl demonio-erG

'A usted lo espantó el demonio'

(12) döb $r$ pirau-jierra-m

licor comprar-pdo.pl-dcl

'Compraron licor'

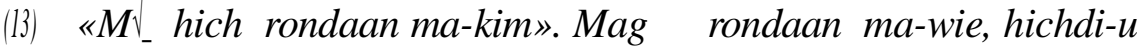
1SG 3SG vigilar ir.prS-inm mientras vigilar ir-ant 3sG-erG hook'a hook'a.

buscar.prs buscar.prs

'«Yo ya lo voy a vigilar». Mientras vigilaba, él busca y busca'

(14) Mua daño hacee-ju-ta

1sG.erG daño hacer-fut-foc

'Yo le haré daño'

(15) $k u) s \quad p \downarrow_{-}$rau-wie, deg $\quad p \downarrow_{-} a p \downarrow_{-} i$-ji-m

puerco agarrar-ant en la casa soltar-pdo.SinG-dc1

'Una vez que había agarrado al cerdo, lo soltó en la cada

(16) Hoo-ba, jöö sereuta, genk'a-wai hõr-aan.

Ver-imp.SinG vayabulla bailar-ant persona-pl

¡Vea, vaya bulla, la gente estaba bailando’

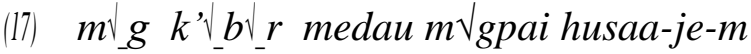

este alquitrán mecha grande usar.prS-hab-dcl

'Se usa esta mecha de alquitrán grande'

(18) hãt'ãrr hãu hichdi-u j\r-baade-ji-m-ta, chi poorpam. gallina huevo $3 \mathrm{~s}$-erG buscar-inc-pdo.SinG-dcl-foc art dañado 'Un huevo de gallina él empezó a buscar, el dañado'

(19) «ip ra-tat, $\quad p \Downarrow_{-}$ra-tat, $\quad p v_{-}$ra-tat!»

Agarrrar-imp.pl agarrar-imp.pl agarrar-imp.pl

'Agarrenlo, agarrenlo, agarrenlo'

(20) Mowee-ba, mowee-ba

Mover-neG mover-neG

'No se mueven, no se mueven' 


\section{Cuadro 4. Sufijos verbales TAM del waunana}

\begin{tabular}{|l|l|}
\hline presente & $\emptyset$ \\
declarativo & $-m$ \\
pasado & $-j i \sim-j i e r r a$ \\
futuro & $-j u$ \\
anterior & - wie $\sim$-wai \\
habitual & $-j e$ \\
imperativo & $-b a \sim-t^{\prime}$ at $(a)$ \\
incoativo & $-b a a d(e)$ \\
negación & $-b a$ \\
\hline
\end{tabular}

Entre los tipos de verbos detectados hay una serie de raíces con valor copular y estativo, distribuidas según distinción de número y tiempo. En cuanto a la de número, las raíces sĩe (21), (22) si (23) $\sim t \_n \_$(24) corresponden al singular y las raíces naa (25) $\sim n \downarrow_{-}$(26) $\sim t \_n a a$ (27) corresponden al plural; en cuanto al tiempo, las raíces si $\sim t \_n \_n \downarrow_{-}$corresponden al presente, mientras que las raíces sĩe $n a a \sim t \backslash$ naa corresponden al pasado. Queda por averiguar si en otras flexiones de TAM ocurre lo mismo, así como aclarar otros detalles sobre el semantismo propio de cada raíz.

(21) di dak'a sĩe-ji-m haa-je-m

casa cerca estar.SinG-pdo-dcl aux-hab-dcl

'(El joven) solía estar cerca de la casa'

(22) jarb $\quad$ sie-ji-m

palo de achiote estar.SinG-pdo-dcl

'Estaba un palo de achiote'

(23) Hichita poorpam si-m-ta

Verdad dañado estar.SinG-dcl-foc

'En verdad está dañado'

(24) chi $h \downarrow_{-}$iran chi hemköi $d \downarrow_{-} i$ pöch $t \downarrow_{-} n \_-m-t a$

art esposa art hombre con envuelto estar.SinG-dcl-foc

'La esposa está envuelta con el hombre' 
(25) jööi-n benk $\sqrt{ }$-n naa-ji-m haa-je-m

viejo-pl brujo-pl ser.pl-pdo-dcl aux-hab-dcl

'Los viejos eran brujos'

(26) döb \\u ma-m fiesta hacee $n \downarrow_{-}-m$

navegar ir.pte-dcl fiesta hacer estar.pl-dcl

'Se va navegando a donde están haciendo la fiesta'

(27) di heegar $t \backslash$ naa-ji-m $k u) s$

casa debajo estar.pl-pdo-dcl cerdo

'Debajo de la casa estaban los cerdos'

Además de esos verbos estativos, es posible encontrar verbos intransitivos (28) (dentro de los cuales están algunos verbos de movimiento (29), (30) y (31)), verbos transitivos (32) y ditransitivos (33).

(28) jöoi $p^{\prime} u b d \sqrt{ }$

viejo brincar.prs

'El viejo brinca'

(29) $\quad M$ _ hich rondaan ma-kim

1SG 3SG vigilar ir-inm

'Ya voy a vigilarlo a él'

(30) Jãk woun-aan huru-m

ese persona-pl venir.prs-dcl

'Esa persona viene'

(31) hewaan bee-wie

joven venir-ant

'El joven había venido'

(32) Jööy-au tööd weeo-wai

viejo-erG toldo abrir-ant

'Cuando el viejo hubo abierto el toldo'

(33) Jööy-ag trago baraaja, hu)skunija(choo) choodee-ba viejo-dat trago no poquito poquito servir servir-neG

'Al viejo no le sirvieron un trago pequeño' 
El orden de los auxiliares es V-AUX. Ejemplo de esto es la perífrasis progresiva en (34). Otra potencial opción es (35). Una tercera forma de perífrasis está relacionada con las cópulas cuyos sujetos son plurales, como se puede ver en (36), (37) y (38). Según los datos, la pluralidad del sujeto obliga al uso de una raíz verbal vacía haa, la cual recibe flexión de habitual y de modo declarativo; por su parte, el verbo manifiesta la flexión de tiempo y modo declarativo. Este fenómeno ha de investigarse con mayor profundidad.

(34) puro flauta $j \downarrow$ rrta $\mathrm{N} \sqrt{ } \sqrt{ }-m$

pura flauta sonar estar.prS-dcl

'Está sonando la pura flauta'

(35) rondaan ma-wie

vigilar ir-ant

'Cuando se hubo ido a vigilar'

(36) $H \backslash$ i- $n$ naa-ji-m haa-je-m t'ãrjup mujer-pl estar.pl-pdo.SinG aux-hab-dcl tres

'Había tres mujeres'

(37) jööi-n benk $\sqrt{-n}$ naa-ji-m haa-je-m

viejo-pl brujo-pl ser.pl-pdo-dcl aux-hab-dcl

'Los viejos eranbrujos'

(38) Celebraanaa-ji-m haa-je-m

celebrar estar.pl-pdo.SinG aux-hab-dcl

'Estaban celebrando'

\section{Adjetivos}

Puede hablarse de adjetivos en waunana, en vista de que los miembros de esta clase de palabras se determinan según dos criterios: semántico y sintáctico. El semántico se refiere a la clase de

13 Aparentemente la progresión también puede expresarse mediante un sufijo $-k$ ' $a$, como se puede ver en (i). Sin embargo hay que indagarlo más con un instrumento independiente.

mag jööi hijejeb-k'am hich meper-ag hijejeb-k'am

anf viejo hablar-proG 3sG demonio-dat hablar-proG

'Ese viejo le está hablando a su demonio' 
carga conceptual que posee este tipo de palabras y el sintáctico se refiere al tipo de estructura en donde se inserta. Hay adjetivos que denotan tamaño, como pum 'grande' y chai 'pequeño'; otros indican estados de ánimo, como meuk'a 'bravo' y meerrau 'enojado', estados físicos hacop'a 'ebrio', tõsin 'borracho', daudau 'palpable, visible', etc. Otros adjetivos son mach 'fuerte', tu)p'ãa 'echado', k'udeergersi 'abrazados', durag 'largo', hippir 'lleno' y poorpam ‘dañado'. La mayoría de las raíces adjetivas son simples, aunque hay casos de adjetivos producto de reduplicación nominal, como daudau 'palpable, visible' (< dau 'ojo') y otros por medio de un mecanismo que ha de ser identificado, como jöingarin 'antiguo' (< jööi- $n$ viejopl 'viejos').

En cuanto al criterio sintáctico, los adjetivos en waunana pueden calificar un sustantivo y ser modificado por otro tipo de morfema, como el diminutivo dam (39); también pueden ser complementos predicativos que afectan a un sujeto (40).

(39) $\mathrm{ku}) \mathrm{s}$ chai dam puerco pequeño dimn 'Chancho pequeñito'

(40) chi hemk'oi $h \downarrow_{-} i_{-} \quad d V_{-} \quad t$ 'u)p'ãwãa $n \downarrow_{-}-m$ art hombremujer con acostado estar.pl-dcl 'E1 hombre y la mujer estaba acostados'

\section{Adverbios}

Al igual que en el caso anterior, los adverbios en waunana pueden identificarse mediante criterios semántico y sintáctico. Según el semántico, los adverbios pueden expresar nociones locativas, como $\operatorname{deg}(a n)$ 'en la casa', dökĩr 'al otro lado', heudee 'atrás', marãk 'río arriba', waurp 'lejos', chum 'allá'; nociones temporales nau 'ahora', warrkur 'antes, anteriormente', hichan 'hoy' y de manera, como haipai 'repetidamente', deeu 'otra vez'. En cuanto al criterio sintáctico, los adverbios pueden calificar a un adjetivo, como se observa en 
(41) y también modificar al verbo, como ocurre en (42). La mayoría son raíces simples; sin embargo, algunos adverbios son versiones locativas de un sustantivo, como $\operatorname{deg}(a n)$ 'en la casa' ( $<d i$ 'casa').

(41)k'udeergersi bit'urgau abrazado de lado 'abrazados de lado'

(42) Jöo, hinanaa $p \downarrow_{-} r \quad t \downarrow_{-} n \downarrow_{-}-m$-ta hagat'a ¡Vaya!, inmóvil agarrado estar.SinG-dcl-foc todavía ‘Vaya!, todavía estaban inmóviles, agarrados’

\section{Adposiciones}

Las adposiciones del waunana son posposiciones. La mayoría de las reconocidas corresponden a situaciones locativas, como hee 'en, dentro' (43) y (44), gai 'en' (45), heegar 'debajo' (46), hiugarbi ‘atrás' (47), jãee 'dentro’ (48), harr ‘donde' (49), jar 'abajo’ (50). También existe una posposición comitativa $d{ }_{-} i$ 'con' (51).

(43) k'ach hee oído en

'Al oído'

(44) do hee agua en

'Dentro del agua'

(45) mag dib $\sqrt{ } g a i$

anf horcón en

'En ese horcón'

(46) di heegar

casa debajo

'Debajo de la casa'

(47) p'etkar hiugarbi

costilla atrás

'Atrás de la costilla' 
(48) di jãee

casa dentro

'Dentro de la casa'

(49) k'aper harr

compañero donde

'Donde el compañero'

(50) $d i \quad$ jar

casa abajo

'casa abajo'

(51) Jööi $\quad h \downarrow_{-} i \quad d \downarrow_{-} i$

viejo mujer con

'Con la mujer del viejo'

Partículas y marcadores de estatus informacional

Dos de los rasgos más sobresalientes del texto analizado dos clases abundantes de elementos abundantes: un gran conjunto de interjecciones, ideófonos y onomatopeyas, por un lado; y el sufijo - $t a$, por otro. En cuanto a las interjecciones, la más común es jöö(k) ‘¡vaya!', y también está \\ ciones hispanas, como ã, aai, ay (jay!). Entre los ideófonos y onomatopeyas presentes se pueden listar los siguientes: chuk chuk (para indicar que agita los huevos), kuwii, kuwii, kurr, kurr, kurr (diferentes voces de los cerdos), dug, dug, dug, dug (para indicar que agita un árbol), keeu, keeu, keeu, keeu (voces de gallinas), ёёii (equivalen-

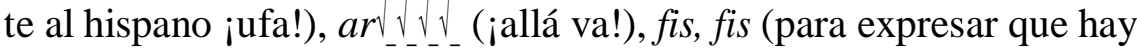
una pelea) y jiii (para indicar susto o sorpresa).

En cuanto a los marcadores de estatus informacional, el waunana posee un sufijo -ta. Según Sánchez y Castro, que cumple la función de marcar participantes o elementos focales, es decir, entidades que se resaltan por encima de otras y a las cuales se debe

14 Sánchez y Castro, 100. 
prestar atención. Los ejemplos (52) y (53) ilustran lo dicho: en (52), el sufijo - ta está añadido a un sujeto, y en (53) a un predicado nominal. Sin embargo, y como se ha visto en los ejemplos anteriores, los elementos oracionales a los que más afecta la afijación de - $t a$, son los verbos, como en bee-ji-m-ta venir-pdo-dcl-foc 'vino'. Es bastante llamativo que el sufijo en cuestión se añada tanto al participante con mayor fuerza argumental como al verbo en una misma oración, como en (52) y (53). También hay evidencia de una marcación con - $t a$ en ideófonos (54) e interrogativos ((64), más abajo). La marcación directa con -ta no es el único mecanismo para realzar participantes, puesto que también se recurre al movimiento de estos al margen izquierdo de la oración. En vista de que en el texto analizado los objetos directos y dativos trasladados a la izquierda no se encuentran marcados con - $t a$, es posible sostener, en esta primera etapa de la investigación, que tal sufijo es un marcador de foco, como lo indican Sánchez y Castro.

(52) ¡Biejo, jãg neper-ta maach haig barbaichin-ta!

Viejo esedemonio-foc $1 \mathrm{pl}$ allí llegar-foc

‘ $i$ Viejo, es ese demonio el que lleGa allí donde nosotros'

(53) iAy, neper daudau-ta si-m-ta!

ay demonio palpable-foc estar.SinG-dcl-foc

'¡Ay, palpable es cómo está el demonio'

(54) chi ku)s-ta kuwii, kuwii sii-ta

art cerdo-foc ideo ideo ideo-foc

'Es el cerdo el que oinc, oinc'

\section{Sintaxis}

Relaciones gramaticales

El waunana presenta un patrón ergativo en la marcación de sus relaciones gramaticales. El sujeto de una oración intransitiva (55)

15 Según Mortensen, la lengua emberá tiene todo un conjunto de sufijos marcadores de foco tanto para el ergativo como para el absolutivo (48-49). 
y el objeto directo de una transitiva (56) no llevan marca alguna, mientras que el sujeto transitivo se marca con el caso ergativo codificado en el sufijo - $u$ (aparentemente, cuando la raíz nominal termina el consonante se agrega una vocal $a$ epentética). También, como en (57), puede que el sujeto no reciba marca de caso. Este sufijo ergativo es homófono con el sufijo instrumental $-u \sim-o$ (59). Otra propiedad del sujeto en waunana es que, como se dijo en el apartado 3.1.2, este establece relaciones de referencia cruzada de número con los sufijos verbales de pasado y de modalidad imperativa. Por su parte, el objeto directo es el sintagma nominal que se encuentra entre el sujeto y el verbo en oraciones transitivas simples (56), (57) y (58), mientras que el dativo (58), marcado con el sufijo - ag, es posverbal.

\section{(55) Magbaadee hemk'ooi mãrmua bee-ji-m-ta}

En ese momento hombre arriba venir-pdo.SinG-dcl-foc

'En ese momento un hombre vino de arriba'

(56)Chi k'aper-au chi nobia hatãrr hau-ji-m

art compañero-erG art novia gallina agarrar-pdo.SinG-dcl

'El compañero agarró la gallina de la novia'

\section{(57) Hewaan numi hatãrr hau-ji-m-ta}

joven dos gallina agarrar-pdo.SinG-dcl-foc

'El joven agarró dos gallinas'

(58)Chipör-au p'atkõn dee-ji-m hich $h \backslash y$-ag

Jefe-erg dinero dar-pdo.SinG-de1 3SG mujer-dat

'El jefe le dio dinero a su mujer'

\section{(59) jööi pegaapai trago-o, do botella-u}

viejo medio pegado.prs trago-ins dos botella-ins

'El viejo se medio emborracha con el trago, con dos botellas'

Orden de las palabras

El orden básico del waunana es SOV para las transitivas y SV para las intransitivas. Tal orden también sirve de modelo para oraciones copulativas como en (53), repetida aquí como (60), en cuyo 
caso el orden es sujeto-predicado nominal-verbo. Si bien existe una tendencia a mantener el orden $\mathrm{OV}$, es posible encontrar objetos a la izquierda de la oración (61) o en posición posverbal (62), cuando hay verbos de dicción y sus objetos son citas (discurso directo). También hay evidencia de dativos a la izquierda (63) y sujetos posverbales (64).

(60) ¡Ay, neper daudau-ta si-m-ta!

ay demonio palpable-foc estar-dcl-foc

‘Ay, el demonio está palpable'

(61)hãt'ãrr ãu hichdi-u j’r-baade-ji-m-ta, chi poorpam gallina huevo 3sG-erG buscar-inc-pdo.SinG-dc1-foc art dañado 'Un huevo de gallina él empezó a buscar, el dañado'

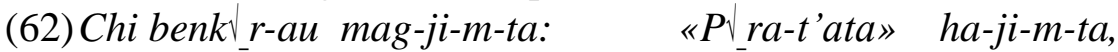
art brujo-erG decir-pdo.SinG-dcl-foc agarrar-imp.pl decir-pdo. SinG-dcl-foc

El brujo dijo «agárrenlo», dijo

(63)Jööy-ag trago baraaja, hu)skunija(choo) choodee-ba viejo-dat trago no poquito poquito servir servir-neG

'Al viejo no le sirven un trago pequeño'

(64) ¿ $p \downarrow_{\_}$, jãga-ta $\quad p \downarrow_{-} \quad j \downarrow_{-} r p \downarrow_{-} i$-ji neper-au?

$2 \mathrm{SG}$ cómo-foc 2SG espantar-pdo.Sing neper-erG

¿AA usted, cómo a usted lo espantó el demonio?'

\section{En el SN}

Los sintagmas nominales están compuestos por un núcleo nominal en forma de sustantivo (65), un pronombre (66), demostrativos (67) u otra clase nominalizada (68). Ese núcleo puede estar acompañado por uno o varios modificadores, entre los cuales están el artículo chi (68), los demostrativos (69), el marcador anafórico mag (70), el diminutivo dam (69), los adjetivos (71), los numerales (72), el cuantificador tönwe (73) y el indefinido haũrag (74). Cuando hay dos adjetivos yuxtapuestos cabe la posibilidad de usar una 
conjunción facultativa naa (73). El orden de los componentes del sintagma nominal no es estricto, puesto que hay numerales posnominales, como en pör numi cabeza dos 'dos cabezas'. La tendencia general es dejar a los cuantificadores, artículo, demostrativos, marcador anafórico y numerales en posición prenominal, y dejar los adjetivos en posición posnominal.

(65) Nepeer daudau si-m

Demonio palpable estar-dcl

'El demonio está palpable'

(66) hamash-diu heerpa $n \downarrow_{-}-m$

ellos-erG mirar estar.pl-dcl

'Ellos están mirando (algo)'

(67) el ultimo, $m \backslash g$ тиа $\quad$ hacee-ju-ta

el último esto 1sG.erg hacer-fut-foc

'el último, esto es lo que yo haré'

(68) Chi mach

art fuerte

'El fuerte'

(69) $M \backslash g$ pendejo dam

ese pendejo dimn

'Este pendejito'

(70) $m \backslash \_-1 n$ mag p'etkar hiugarbi hausiu-wie

este-pl anf costilla atrás dejar-ant

'Dejó (el huevo podrido) detrás de la costilla de estos'

(71) Hemk'ooi hewaan

hombre joven

'El hombre joven'

(72) Hewaan numi hatãrr hau-ji-m-ta

joven dos gallina agarrar-pdo.SinG-dcl-foc

'El joven agarró dos gallinas' 
(73)Tönwe jãk- $\_\_$jayap p'akõtõ (naa) nempum

Todo esa-pl cuatro vaca gorda conj grande

'Todas esas cuatro vacas gordas y grandes'

(74) Haũrag jãk- $n$ hua hãp $m \downarrow_{-}$chaai- $n j \backslash p^{\prime} \downarrow_{-} r$

Alguno ese-pl cinco 1sG niño-pl flaco

'Alguno de aquellos cinco niños flacos míos'

En cuanto a la posesión, se han detectado dos formas: una, mediante el orden poseedor-poseído (75); la otra, con el mismo orden pero incluyendo el morfema de posesión din para marcar el poseedor (76). Los ejemplos encontrados no dan una idea clara de las posibles diferencias de sentido entre estas dos formas (ambos son partes del cuerpo, por lo que, al menos hasta que no hayan nuevos datos, la diferencia entre posesión inherente y no inherente queda descartada). Otro aspecto destacable es que los ejemplos (77) y (78) señalan que la lengua waunana muestra el fenómeno de la frase nominal escindida. En (77) se muestra una escisión en el numeral y en (78) existe una escisión en el numeral y el demostrativo. Aparentemente, el numeral permite arrastrar al demostrativo, puesto que un demostrativo solo en posición posverbal no lo aceptan los hablantes.

\section{(75) $k u) s \quad$ büch $\_n$} cerdo barriga 'La panza del chancho'

(76) $c h i \quad h i_{-}$din p'etk'ar

art mujer pos costilla

'La costilla de la mujer'

(77) $H \downarrow$ 느- $n \quad$ naa-ji-m haa-je-m t'ãrjup

mujer-pl estar-pdo.SinG aux-hab-dcl tres

'Había tres mujeres'

(78) $N o \downarrow_{-} \mathrm{i}-n \quad h a-j i-m-t a \quad m \downarrow_{-} \mathrm{k}-\downarrow_{-} n$ t'ãrjup

novia-pl tener-pdo.SinG-dcl-foc este-pl tres

'Tenía tres novias' 
En el SV

Un sintagma verbal puede estar compuesto por un núcleo verbal simple (79) o con auxiliar (80). A este núcleo verbal se le puede añadir, según lo visto, un objeto directo y un dativo. También es posible la presencia de adjuntos oblicuos con un grado de libertad estructural mayor, pues los hay tanto preverbales,(81) y (82), como posverbales, (82). No obstante, la tendencia es que el verbo sea el elemento final. También se ha encontrado un intensificador, nem, el cual es preverbal (83).

(79) «ip $\downarrow_{-}$ra-tat, $\quad p \downarrow_{-}$ra-tat, $\quad p \downarrow_{-}$ra-tat!» agarrrar-imp.pl agarrar-imp.pl agarrar-imp.pl

'Agarrenlo, agarrenlo, agarrenlo'

(80)fiesta hacee naa-ji-m haa-je-m

fiesta hacer estar-pdo-dcl aux-hab-dcl

'Estaban haciendo una fiesta'

(81) deg jupima-wia

en la casa sentarse-ant

'Cuando se sentaron en la casa'

(82) tood hee $p \backslash$ rba-baad di jar

toldo en dar vuelta-inc casa abajo

'Empieza a dar vueltas en el toldo, abajo de la casa'

(83) Hewãan nem kãi-tar jau-kim

joven intS dormir-nmlz decir-inm

'Ya voy a contar (la historia de) el joven que tenía muchas ganas de dormir (con una mujer)'

\section{Conclusión}

Se ha mostrado un panorama general de aspectos sobre la morfología y la sintaxis de la lengua waunana. Por tratarse de una primera aproximación, queda la sensación de que todavía hay asuntos por resolver o que quedan sujetas a revisión. Es necesario profundizar 
en la morfología derivativa, tanto nominal como verbal y también en lo referente a otras categorías menores, como los nexos oraciones y los marcadores de discurso. Además, queda pendiente más investigación sobre procesos sintácticos, de la subordinación completiva y relativa, y de la diátesis.

\section{Abreviaturas}

$\begin{array}{llll}1,2,3 & \text { persona gramatical } & \text { imp } & \text { imperativo } \\ \text { anf } & \text { anafórico } & \text { inc } & \text { incoativo } \\ \text { ant } & \text { anterior } & \text { incl } & \text { inclusivo } \\ \text { art } & \text { artículo } & \text { inm } & \text { inminencial } \\ \text { aux } & \text { auxiliar } & \text { ins } & \text { instrumental } \\ \text { conj } & \text { conjunción } & \text { intS } & \text { intensificador } \\ \text { dat } & \text { dativo } & \text { neG } & \text { negación } \\ \text { dc1 } & \text { declarativo } & \text { nmlz } & \text { nominalizador } \\ \text { erG } & \text { ergativo } & \text { pdo } & \text { pasado } \\ \text { exc1 } & \text { exclusivo } & \text { pl } & \text { plural } \\ \text { foc } & \text { foco } & \text { poS } & \text { poseedor } \\ \text { fut } & \text { futuro } & \text { prS } & \text { presente } \\ \text { hab } & \text { habitual } & \text { SinG } & \text { singular }\end{array}$




\section{Historia tradicional}

\section{Hewan nem kãitar}

Hewan nem kãitar jaukim. Maat'a $h \sqrt{ }$ in naajim, haajem, t'ãrjup. Magbaawai, hãbakai di dak'a siejim, haajem. Hemk'ooi hewan. Mag no $\_$in hajimta mak $\sqrt{ }$ t'ârjup. Magbaadee hemk'ooi mãrmua beejimta hewan beewie. Magbadeewai chi hewan k'euraa beewie magjimta: “Jööi $h \downarrow_{\_} i d \_i$ kãijuta”. Magbadee habak'ayiu hãk'a sim dinaawai, hichdeu meermajimta meraa nem k'aibaadewie hedar. Petaadijim di taag waid $\bigvee_{\text {baad dib }} \bigvee_{\text {gai, mag dib }} \bigvee_{\text {gai waid }} \bigvee_{\text {wie, }}$ magbaadee hiriwan hich k'aper hich haig k'aiju, piiajimta. Hewaan numi hatãrr haujimta. Chi k'aperau chi nobia hatãrr haujim. Magjimta: "Jöök, mag pendejon. $M \backslash$ noo $i$ hatãrr hatamta. $M \downarrow$ hich rondaan makim”. Mag rondaan ma-wie, hichdiu hook'a hook'a. Chi noo _iag charcha hook'awie. Tööd huk'arii tööd weeo, hoowie chi hemk'oi $h \backslash i d \downarrow$ i $t^{\prime}$ 'u)p'ãwãa $n \downarrow-m$ lo do k'udeergersi bit'urgau. Jöök, hich hudeg pai magjim han $\sqrt{ } m:$ : $M \backslash g$ pendejo dam. Миа hi daño haceejuta, тиа daño haceejuta". Magbadeewai mag-jim-ta: " $m \_g$ jööi jööinata, jööin benk $\sqrt{ } n$ hajim", hajem. Magdeewai di heegar $t \backslash$ naajim ku)si, $t \backslash n a a-$ ji-m hãt'ãrraan. Magbaadeewai hichdiu magjimta: "Hãt'ãrraan jöingarin, hãt'ãrraan, hãt'ãrraan di heegarpai hãu j】_ch piejejimta hakö̋l_i. Magbaadeewai hãt'ârr hãu hichdiu j\_rbaadejimta, chi poorpam. Magbadee hichdiu k'ach hee mag "chuk chuk, a chuk chuk sim" hajim. "Hichita poorpam si-m-ta". Magbaadee hich-diu nemãu pör numi. $M \backslash$ g pö̈ numi hauwie. Magbaadeewai, $m \downarrow g \downarrow$ n $\operatorname{mag}$ p'etkar hiugarbi hausiuwie. Himagua chi $h \_$i din p'etk'ar garju haku hausiujim chi nemãu chi pör hat'arrk $n$. Magbaadeewai, chi wounaan, chi he wanaan petarrau di heegar ku)s tu)p'ãa $t \sqrt{ } n \backslash m t a . K u) s$ tag ku)s tup $p^{\prime a ̃ a}$ $t \downarrow$ naawai, magua chi hẽwanau ku)s bich $\_n$ dinnuk'ap $\downarrow$ _bajem, $\_r \_r l g$ sii di hegar dichdi-baad, jöö, \_r_rıg dichdi-baad. Magbaadee chi ku) sta kuwii, kuwii siita kurr, kurr, kurr siita. Chi ku)s regaap'ubaad. Magbaadee chi wounaan chi brujo hai siejimta. Chi bẽnk $\bigvee$ )rau, mag 
chi bẽnkฟ)rau magjimta: “Jöö” Hich huan p'iid Vbaadejimta, hich hu) anjã hakö". Mag hich hu)an p'iid Vbaadewie: "Haai, biejo, jag neperta maach haig barbaichinta. Magbaawai fiesta haajim hagmãrag. Hagmarãgaa siejim como un calle fiesta hacee naajim hajem. Maigmua celebraa naajim haajem. Magua hichappai $m \_g$ jööi mam bagar di wai sierrawai, mam hich mam siejim. Magbaadeewai ya $m \backslash \mathrm{g}$ $k^{\prime}$ u)s p'iid tk'abaadeewai ya neper hajimta. Jöö, chi benkl_rau magjimta: "P\ra-tat" haajimta, " $\bigvee_{\text {ratat }}$ " haajimta. Keeo. Magbaadeewai chi ku)s calmaak'abaadeewai; $n \backslash \downarrow \downarrow$ _ deeu chi kuls

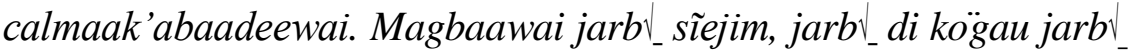
$m \downarrow$ g gaai hãt'ãrraan sii hãt'ãrr kapan k'ai $n \backslash \_$dinawai. Magbaawai chi hemk'ö̈au pet'arrau chi jarb gaai. Dug, dug, dug, dug, habarwai chi hãt'arr k'ar hĩerrau chi hãt'ãrr chi hãt'ãrr hierrau chi sĩiöörp'ubaad keeu, keeu, keeu, keeu. Jöö, magjim han \m. Hich hu)anãu magjim han $\_$m: "Biejo", haajim han $\_m:$ "Aai”, hajim han $\_$, "neperaan daudau simta. $M \backslash k \backslash n$ neper daudau beeta $m \backslash$ ingin daudauta haadimta". Jöö. Magwaiyi chi $h \backslash$ iran chi hemk'ö̈ $d \backslash \_$i pö̈h $t \_n \_m t a$. Hagt'a hag $d \_i$ up $r$ $t \geqq n \_$mta hagat'a. Moweeba, moweeba. Jöö, hinanaa $p \backslash r t \_n \_m t a$ hagat'a. Bueno, ya mak $\_n$, mag jööi hijejebk'am hich neperag hijejebk'am: “ip $\Downarrow_{\text {ra-tat, }} p \backslash$ ra-tat, $p \backslash$ ra-tat!”. Keewa. Wounawai jãga p\rauju, bibo $n \downarrow_{-} \eta_{\_} m$ hawai. Jöök. Magbaadee, hichidiu magjim: "el ultimo, m\g тиа hacee-ju-ta. Hichdiu hĩu waisijim ku)s kuls chaain dam, pum ka-ba kit _-m kuls chaain dam. Magbaawai ku)s $p \downarrow$ rauwie, deg $p \downarrow$ _ap $\backslash$ _ijim deg $p \downarrow$ _ap $\backslash$ _wie chi ku)s $b \downarrow$,

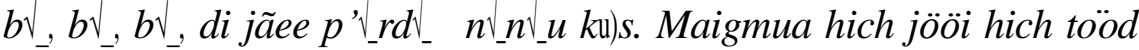
heетиа жеоо $n \backslash m$. "Ay, bieja. Ku)s k'a deeu wë̈ $\_$_m” haajim. "Ku) s, jag ku)s neperau j $\backslash$ rap $\backslash$ ijim degag”. Jöö, hich jööi hijeeb $\downarrow$ _t_ naajim hich jööi neperag neper: " $p v_{\text {ra-t }}$ 'at, $p p_{-}$ra-t'at, $p v_{-}$ra-t'at”. Bueno mag sim chi woun nibãrjakãijã piid $\_$piid $\_$juga; jöo"hagat höp $\_r \mid n \_n \_m$. Magwie, magbadeewai el ultimo magjim jãgaa barekim m\g jööiag köpurta $p \backslash$ rk'ajuta köpurr. P\_k'awie kolaao hee, kolaau hee hiipir

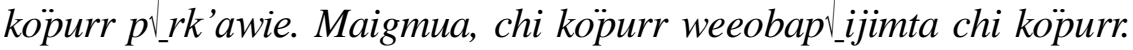




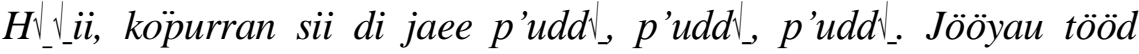
weeowai, köpurr di jãee ya köpurr jĩerrau köpurran. Magbaadewaita, hichdiu chi dauper tööd weeo $n \backslash \_$jim. Hëëii nemdaumigtokgan $\downarrow \_t, t_{-}$ di jãee töödan tood p'ut sii. Magbaadeewai, chi hemk'ooi sii tööd hee

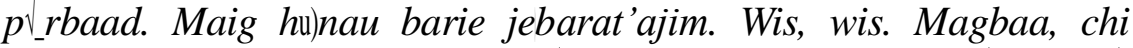

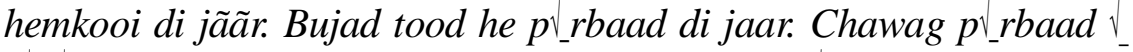
$r \_r \_g$, petawie dö hee ;chunbad! Pendejo beraa $t \backslash$ naawie badgajar , p'usa hubir baima hich k'aper harr. Maigmua hich k'aper harr ubir baimawie magjim han $n:$ : "ay", haajim han $\_n$. "Nen cholo, $p \downarrow_{-}$ k'anitoogan hajim. ¿Kanjakwie $p \downarrow$ _k'amiiu si? Jukkaa, domer hap $i$, haajim. Jöö, hichan $p \downarrow_{-} m \downarrow_{-} d \_i$ kãibam”, hajim. Magbaadee, pendejo dam, pendejo dam, hich k'aper haup $\vee_{-}$jim. "Ya $p v_{-} m \downarrow_{-} d \_i$ k'aibam", habaawai. Hich jap chaai dam hauwie, hich jap dökĩr dı_rbajim. ¡Ar \\\\! Magua habak'anu)a hakju heudee petajim, hakju marãk döbööu majim fiesta hacee $n \backslash m$ harig. Warrkur mechon $m \sqrt{ }$ gpai husaajerrawai, mechon dam ha $n \backslash m, \quad k^{\prime} \backslash b v_{-} r m \_g \quad k^{\prime} \backslash b \backslash r$ medau

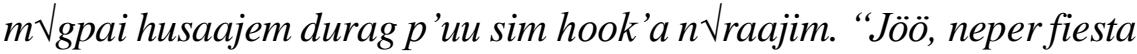
haawaik'aba”, haajim. Neper daudau sim. Magbadee habak'ayiu, chi

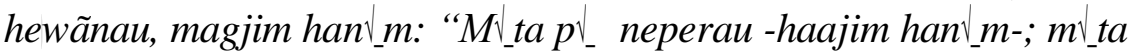
$p \downarrow$ neperau” haajim. Jööu, magjim habarm\n jööyau "karau”, jööyau "karau chaai dam $p \downarrow$ gai mиа maswaup $\downarrow$ yaag pam “ hajim han $\backslash$ _m.

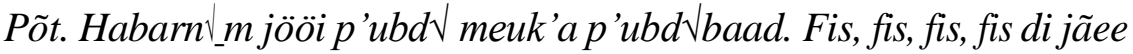
fees chirt $\sqrt{ }$ haaukansii. Hewãnau magsiewia p'ubd $\sqrt{ }$ baadem, hewãnau ju) $r$ fis, fis; naawie jööi pum benk $\downarrow \_r$ di beeug deejim $d \backslash$ iichagju. Magbaa chi $h \backslash$ in: "Haay, ¿pãar kanteet'a jãup'ubad? Al fin, magbaa chi $\mathrm{h} \sqrt{ }$ _inau kecheu barata. Jöö, hichab jööi hakop'a sierra, kãibaadejimta. (Warrgar $\sqrt{ } n k \sqrt{ }$ nan pua $k$ 'ap $\ n$ $m \downarrow g$ di jaana töön harruta täjerrawai.) Magbadee, hich jööi k'aisiiawie, jööi p’igd baad, hajuata juajerrawai. Magbaawia w_rkig hai siejim haig $h \backslash) u t \bar{\downarrow}$ $\mathrm{h} \downarrow) u t \_$maimua haig nampiiauwie. Magbaa chi hewan deg waigd $\downarrow$ di keeu gaai, jupbaimajimta jag $\downarrow$ n di keu haar. Magbaawai hegeu hegeu hawie mansii kiut _ di kierma, mag chi di kier kiut _ kiut _ sim hawai. 
Magbaawai, hãju)ataju)a sim dinaawai. Magbaa hich mekeu mag wöö simee p'îiat'a. Magbaawai, porkeria chaayau [jãumamua] mag chi

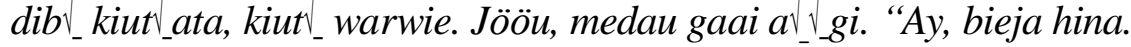
¿Jãmpai döö heepaji?” chi hemkooi hook'awai. Magwai ya waurp hiriup'ubaad chum hiriup'ubaad $p$ '﹎ord. Mamagap mawie, mag $d o b \downarrow_{\_} r$ piraujierram boteed $\downarrow_{-} j a p ;$ döb $\downarrow_{-}$p pirau-wie, hamachdiu heerpa $n \downarrow$ m. Jöö, ya jãg, jãg ya nepe-n $j_{\_} r p \_$itarr hawai. "Jãk wounaan hurum” haajim. Chagcha döö hee hoowai, badag heerpawai döö higau marag p'uun _ chi lampara marag hurum han $\_m$. "Nau maachdiu hirig jeuju”, haajim. “„Nau jãgat'a jãk woun uru?”. Jãk woun mag hurum, hurum, hurum hamaig $p$ '﹎. riu jopbaichi jer, jap

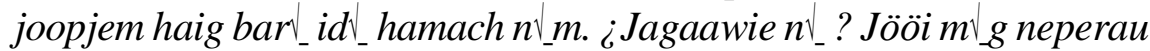

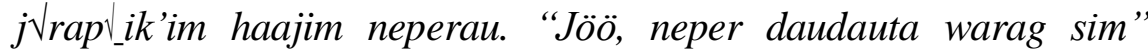
haajim. "Jöö, ay tío duba -haajim trago. Jööiyag trago baraaja, hu) chkunija choodeeba. Warkurim trago jarr pör tãu pör hee hippir chi mach döpiejeejim. Jöö, haipai dudu naawie haigpai siiubaad. "Ay tío,

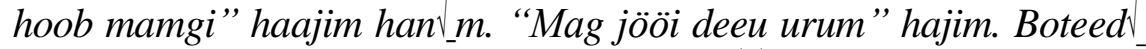
jöӧуаи deeu; magbaadee deeu jujupdu hiy\__. "Jöö, chi dauperaan maach deg pauk'abaadem”. Maagwaiyin degan musica j`rrta $\mathrm{t} \sqrt{ } \sqrt{ }$ m, puro flauta j\_rrta $t \sqrt{ } n \sqrt{ }$. Hooba jöo“sereuta jemk'awai hõõr-an. Jöö mamag siiewie, jööi pegaapai tragoo, do botellau pegaapaiaadejim. Magbadee hamach deg pauk'ap'ubaad. Jöö, deg jupimawie, hich jööi

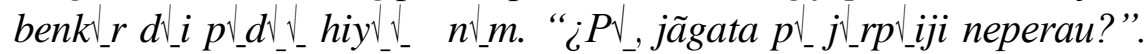

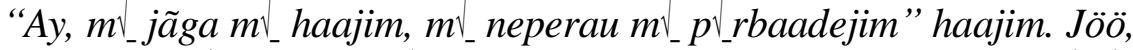
magbaadem $\downarrow$ n, ëëi, chi h〉_yau jürk'aowai sii me pa puunata p'us $t \downarrow_{-} n \downarrow_{-}$ nta. Jöö, ay јӧӧуаи herpajim hawantabaabawai hichdiu jeuatajim.

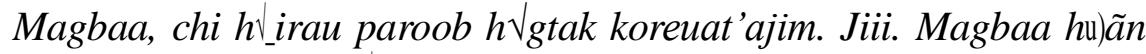
jua dam hee chuibap $\_i$ jööi dinan. Jööu maigta hõõr hiuk'a naajim. "¡Ay, neper daudauta simta!” Magbaa hich jööi meerrau "¡karau!”. Jööi tõsin dinaawai meerrau “ip\_rat'at!” Jöö, magbawie, jöömagwie hich jööi hideu k'aipiba. Maipapai chi cuento ya. 


\section{Traducción}

\section{El joven que durmió con una mujer}

Voy a contar sobre el joven que durmió con una mujer. Resulta que había tres mujeres. Entonces un hombre estaba cerca de la casa. Era joven y tenía tres novias. El hombre venía de arriba. El joven vino en la tardecita. Dijo: «Voy a dormir con la mujer del viejo». Entonces otro hombre se puso celoso, y como estaba celoso, se fue escondido sin acostarse con su novia la misma noche. El que quería dormir con la mujer del viejo subió a la casa. Él pensó entonces que el otro iba a dormir. Eran dos jóvenes.

El que tenía tres novias robó una gallina. El otro dijo: «Vaya, este pendejo robó la gallina de mi novia. Me iré a vigilar». Y se fue a vigilar. Este miró, miró a su novia verdadera, buscando dentro de la casa sin ser notado. Abrió el toldo y otro hombre estaba acostado con la novia de él. Pensó: «Le voy a hacer daño a este pendejo, le voy a hacer daño». El dueño de la casa era anciano y brujo. Debajo de la casa había chanchos y gallinas. Entonces, el que abrió el toldo dijo: «Voy a coger un huevo» (antiguamente las gallinas ponían huevos debajo de la casa). Entonces, él buscó los huevos dañados. Empezó a mover los huevos, chuc, chuc, chuc, chuc, y encontró uno dañado. Entonces cogió dos huevos dañados y los dejó atrás de la espalda del hombre que dormía con su novia. También atrás de la espalda de la mujer. Entonces el hombre joven fue debajo de la casa donde estaban los puercos acostados. Pisó las barrigas de los chanchos. Los chanchos se espantaron y se fueron todos. Entonces los puercos chillaban por otro lado. El hombre le dijo al brujo: «iVaya, lo levantó la vieja!» Entonces la vieja dijo: «iAy, viejo. Ese es el demonio que ha llegado». En ese momento había una fiesta más arriba. Estaban celebrando. Como el viejo tenía su casa abajo, entonces los chanchos se fueron. Esto fue por el demonio. « $i$ Vaya», dijo el viejo. «Agárrenlo, agárrenlo». Entonces los puercos se calmaron. 
Había un árbol de achiote. En ese árbol estaban durmiendo bastantes gallinas. El hombre se fue al árbol de achiote y lo movía y movía y las gallinas se regaron por todos lados. Este hombre dijo: «Vaya». La vieja dijo: «Viejo, el demonio está palpable, vaya!» Entonces la novia del hombre todavía estaba acostada con su amante sin moverse. En eso, el brujo estaba hablando con su demonio: «Agárrenlo, agárrenlo, agárrenlo». Pero nada. Como es persona, ¡cómo lo van a agarrar! Entonces él dijo así: «Voy a hacer esto». El brujo buscó un chanchito y lo metió en la casa. Cuando lo metió en la casa, el puerco daba vueltas y vueltas dentro de la casa. Entonces el viejo abrió su toldo y dijo «Ay, vieja, el puerco está caminando dentro de la casa. Ese puerco es un demonio». La vieja dijo: «Échalo para afuera». Entonces el viejo empezó a hablarle a su demonio. «Agárrenlo, agárrenlo, agárrenlo». Mientras esto pasaba, el otro hombre todavía estaba acostado con la muchacha.

El hombre que pisó los chanchos dijo: «Voy a hacer esto. Voy a recoger sapos en un motete lleno». Los recogió y los vació en la casa. Los viejos gritaron: «Ay, los sapos están dentro de la casa. Saltan, saltan, saltan por todos lados». Entonces, el que soltó los sapos abrió el toldo de la muchacha y gritó: « ¿Ufa, toda la casa está hedionda!». Entonces el hombre que estaba dormido se quedó envuelto todavía y la vieja sacó una varilla y le dio rejo. El hombre dio vueltas, se cayó y se fue corriendo al agua y se metió. Salió río abajo donde estaba un amigo. El amigo dijo: «Usted está hediondo, ¿por qué está así? Vaya a bañarse rápido. No vamos a dormir juntos». Entonces el pendejo, el que dijo eso, lo dejó. Se fue en bote al otro lado. El otro se fue atrás porque había una fiesta arriba.

El que se fue primero llegó a la casa, prendió la lámpara y se fue arriba.

«Porque es la fiesta del diablo es que este anda palpable», dijo el viejo brujo. El otro joven dijo: «Yo soy tu demonio». Entonces, cuando dijo esto, el viejito dijo: «Carajo, carajo. Te voy a pegar». Y lo golpeó. Ellos se pelearon dentro de la casa. El joven tumbó al viejo 
brujo también. Entonces las mujeres dijeron: «¿Por qué pelean?», y los apartaron. Como el viejo estaba bien borracho se durmió.

Las antiguas casas fueron hechas con tablas de arr, que eran fuertes. Entonces el viejo se levantó otra vez. Como en su tiempo ellos se ponían taparrabo, el viejo buscó un lugar abierto entre las tablas y se fue moviendo. Entretanto, el joven estaba sentado a la orilla de la casa. Corrió las tablas y vino el viejo para orinar en el hueco que el joven dejó abierto. El viejo metió su pene en ese hueco y el joven lo cerró. El viejo empezó a gritar: «Ay, vieja, dónde fue el hombre», pero el hombre ya se había ido lejos y llegó a su casa.

Los hombres compraron licor, una botella. Cuando vieron para arriba, venía una persona bajando. «Seguramente ese hombre fue echado por el demonio», dijeron. Al ver para abajo, había una lámpara prendida que venía para arriba. Ellos dijeron: «Vamos a preguntarle cómo viene», así. La lámpara se acercaba y llegó al puerto. «¿Por qué el demonio echó al viejo? El diablo está palpable. ¡Ay, tío. Tome un trago», le dijeron al de la lámpara. A este tío le dieron un buen trago. Todos estaban tomando y tomando y se acabó. Los dos hombres dijeron: «Ah, tío. Todavía no se vaya». El viejo compró otra botella y se sentaron conversando y dijeron: «Llegaron los muchachos a nuestra casa» y empezaron a tocar música. Pura flauta. Había mucha gente bailando. El viejo estaba bien borracho con los tragos y estaba hablando con un brujo, preguntando algo. «¿Qué me pasa, que el demonio me está atacando?». La mujer de este borracho empezó a tocarle el pene. Cuando lo tocó estaba erecto. El viejo no aguantó. La mujer, sin querer lo tocó y lo levantó, y dijo: «Uy». Entonces el viejo echó el semen en la mano de la vieja y la gente se reía. «Ahí queda el demonio. Está palpable». Entonces el viejo estaba regañando a su demonio y a la vez estaba borracho. Decía: «Agárrenlo». La gente decía: «Déjalo que duerma». Hasta ahí nomás el cuento. 